\title{
Long Noncoding RNA HEIH Promotes Colorectal Cancer Tumorigenesis via Counteracting miR-939-Mediated Transcriptional Repression of Bcl-xL
}

\section{Chunhui Cui, MD \\ Duanyang Zhai, MS \\ Lianxu Cai, MS \\ Qiaobin Duan, MS \\ Lang Xie, MS \\ Jinlong Yu, MD}

Department of General Surgery, Zhuijing Hospital, Southern Medical University, Guangzhou, China

\begin{abstract}
Purpose
Studies have found that long noncoding RNA HEIH (IncRNA-HEIH) is upregulated and facilitates hepatocellular carcinoma tumor growth. However, its clinical significances, roles, and action mechanism in colorectal cancer (CRC) remains unidentified.
\end{abstract}

\section{Materials and Methods}

IncRNA-HEIH expression in CRC tissues and cell lines was measured by quantitative realtime polymerase chain reaction. Cell Counting Kit-8, ethynyl deoxyuridine incorporation assay, terminal deoxynucleotidyl transferase dUTP nick end labeling staining, and nude mice xenografts assays were performed to investigate the roles of IncRNA-HEIH. RNA pull-down, RNA immunoprecipitation, chromatin immunoprecipitation, and luciferase reporter assays were performed to investigate the action mechanisms of IncRNA-HEIH.

\section{Results}

In this study, we found that IncRNA-HEIH is significantly increased in CRC tissues and cell lines. IncRNA-HEIH expression is positively associated with tumor size, invasion depth, and poor prognosis of CRC patients. Enhanced expression of IncRNA-HEIH promotes CRC cell proliferation and decreases apoptosis in vitro, and promotes CRC tumor growth in vivo. Whereas knockdown of IncRNA-HEIH inhibits CRC cell proliferation and induces apoptosis in vitro, and suppresses CRC tumor growth in vivo. Mechanistically, IncRNA-HEIH physically binds to miR-939. The interaction between IncRNA-HEIH and miR-939 damages the binding between miR-939 and nuclear factor $\mathrm{kB}(\mathrm{NF}-\mathrm{kB})$, increases the binding of NF-KB to Bcl-xL promoter, and promotes the transcription and expression of $\mathrm{Bcl}-\mathrm{xL}$. Moreover, $\mathrm{Bcl}-\mathrm{xL}$ expression is positively associated with IncRNA-HEIH in CRC tissues. Blocking the interaction between IncRNA-HEIH and miR-939 abolishes the effects of IncRNA-HEIH on CRC tumorigenesis.

\section{Conclusion}

This study demonstrated that IncRNA-HEIH promotes CRC tumorigenesis through counteracting miR-939-mediated transcriptional repression of Bcl-xL, and suggested that IncRNA$\mathrm{HEIH}$ may serve as a prognostic biomarker and therapeutic target for CRC.

Southern Medical University, 253 Industrial

Avenue, Guangzhou 510282, China

Tel: 86-020-61643213

Fax: 86-020-61643213

E-mail:jinlonggyu@163.com

Received May 11, 2017

Accepted October 27, 2017

Published Online October 30, 2017

\section{Key words}

Long noncoding RNA, Colorectal neoplasms, Tumorigenesis, miR-939, NF-KB, Bcl-xL

\section{Introduction}

Arising from the transformation of colon or rectum epithelial cells, colorectal cancer (CRC) is one of the most common malignancies worldwide and causes about 694,000 deaths every year [1]. During the initiation and progression of CRC, multiple germline and somatic mutations accumulated, particular for some critical oncogenes and tumor suppressors $[2,3]$. With great advances of the understanding of CRC pathogenesis, novel therapeutic strategies have been developed and greatly improved CRC patients' prognosis, such as 
the molecularly targeted agents and immunotherapy [4]. However, the molecular mechanisms underlying CRC tumorigenesis are complex and many different driving events contribute to these processes $[5,6]$. Further revealing the underlying molecular mechanisms and developing more efficient therapeutic interventions could significantly improve the outcome of CRC patients.

Accumulating genomic and transcriptomic sequencing results have uncovered that only small proportion of the human genome is transcribed into protein-coding mRNAs, whereas the majority of the genome is transcribed into noncoding RNAs (ncRNAs) [7]. Among the ncRNAs, long noncoding RNA (lncRNA) is a class of novel identified ncRNAs with more than 200 nucleotides in length [8-10]. Recent evidences have revealed that lncRNAs have important roles in various pathophysiological processes, and are frequently deregulated in many diseases, particular in cancers $[11,12]$. IncRNA-HEIH has been reported to be upregulated in hepatocellular carcinoma (HCC) and facilitates HCC growth [13]. However, the clinical significances and roles of lncRNA$\mathrm{HEIH}$ in CRC are still unknown.

MicroRNA (miRNA) is another class of functionally important ncRNAs with 21-25 nucleotides in length [14,15]. miRNAs have important roles in the regulation of gene expression via binding with mRNAs at complementary sites, and then inducing target mRNAs degradation and/or translational repression [16,17]. Except the well-known roles of miRNAs in mRNAs, several reports have shown that miRNAs directly modulate gene transcription via binding to promoters, enhancers, or transcription factors [18]. In our previous study, we have found that miR-939 physically binds to transcription factor nuclear factor $\kappa \mathrm{B}(\mathrm{NF}-\kappa \mathrm{B})$, prevents the binding of NF- $\kappa \mathrm{B}$ to the promoter of NF- $\kappa \mathrm{B}$ target gene $\mathrm{Bcl}-\mathrm{xL}$, inhibits $\mathrm{Bcl}-\mathrm{xL}$ transcription, and induces cell death [19]. Intriguingly, using bioinformatics analysis, we found three miR-939 binding sites on lncRNA-HEIH. Therefore, in this study, we further explored the interaction between lncRNA-HEIH, miR-939, NF- $\mathrm{B}$, and Bcl-xL. Moreover, we measured lncRNA-HEIH expression in CRC tissues and cell lines, analyzed its association with clinicopathological characteristics and prognoses of CRC patients. Using in vitro and in vivo functional assays, we investigated the roles of lncRNA-HEIH in CRC tumorigenesis. The contribution of the interaction between lncRNA-HEIH and miR-939 to the roles of lncRNA-HEIH were also detected.

\section{Materials and Methods}

\section{Tissue specimens}

A total of 84 paired CRC and adjacent normal mucosa tissues were obtained with informed consent from CRC patients who had received surgery at Zhujiang Hospital, Southern Medical University (Guangzhou, Guangdong, China). All the tissues were pathological diagnosed by the Department of Pathology, Zhujiang Hospital. The fresh tissue specimens were immediately frozen in liquid nitrogen until use.

\section{Cell lines and cell culture}

The human colon normal epithelial cell line NCM460 and CRC cell lines HT-29, SW480, HCT116, and LoVo were obtained from the Cell Bank of the Chinese Academy of Sciences (Shanghai, China). NCM460 was maintained in Dulbecco's modified Eagle's medium (Invitrogen, Carlsbad, CA). HT-29 and HCT116 were maintained in McCoy's 5A Medium (Sigma-Aldrich, St. Louis, MO). SW480 was maintained in L-15 Medium (Invitrogen). LoVo was maintained in Ham's F-12K Medium (Invitrogen). All the cells were grown in the above described medium supplemented with $10 \%$ fetal bovine serum (Invitrogen) at $37^{\circ} \mathrm{C}$ in a humidified atmosphere with $5 \% \mathrm{CO}_{2}$.

\section{RNA extraction and quantitative real-time polymerase chain reaction}

Total RNA was extracted from frozen tissues and culturing cells with the TRIzol reagent (Invitrogen) in accordance with the manufacturer's protocol. Reverse transcription was performed with the M-MLV Reverse Transcriptase (Invitrogen) to produce the first strand cDNA. Quantitative real-time polymerase chain reaction (qRT-PCR) was carried out with the SYBR Premix Ex Taq II (Takara, Dalian, China) on an ABI 7500 Real-Time PCR System (Applied Biosystems, Foster City, CA) in accordance with the manufacturer's instructions. glyceraldehyde 3-phosphate dehydrogenase (GAPDH) was used as an endogenous control for lncRNA and mRNA. The primers used are as follows: for lncRNA-HEIH, 5'-CCTCTTGTGCCCCTTTCT-3' (forward) and 5'-AGGTCTCATGGCTTCTCG-3' (reverse); for Bcl-xL, 5'-CGTGGAAAGCGTAGACAAG-3' (forward) and 5'-AAGAGTGAGCCCAGCAGAA -3' (reverse); and for GAPDH, 5'-GGTCTCCTCTGACTTCAACA-3' (forward) and 5'-GTGAGGGTCTCTCTCTTCCT-3' (reverse). The expression of miR-939 and pri-miR-939 was measured by qRT-PCR as above described, using TaqMan MicroRNA Assay (Applied Biosystems) and TaqMan PrimiRNA Assay (Applied Biosystems) respectively, in accor- 
dance with the manufacturer's protocols. The expression of target genes was calculated using the comparative $\mathrm{Ct}$ method.

\section{Construction of vectors}

Full length transcript of lncRNA-HEIH was polymerase chain reaction (PCR) amplified from cDNA using PrimeSTAR HS DNA polymerase (Takara) with the primers 5 'GGGGTACCGTCCCCGCCCCCTGC-3' (forward) and 5'CGGGATCCCAAGGTTGGAAAATCCCACTTT-3' (reverse). The PCR amplified product was inserted into the Kpn I and BamH I sites of pcDNA3.1 (Invitrogen) and pSPT19 (Roche, Mannheim, Germany) vector, termed pcDNA3.1-HEIH and pSPT19-HEIH, respectively. The pcDNA3.1-HEIH and pSPT19-HEIH with mutations in miR-939 binding sites was synthesized by GenScript (Nanjing, China) and termed pcDNA3.1-HEIH-mut and pSPT19-HEIH-mut, respectively. $\mathrm{Bcl}-\mathrm{xL}$ promoter region containing the NF- $\mathrm{kB}$ binding sites was PCR amplified from genomic DNA using PrimeSTAR HS DNA polymerase (Takara) with the primers 5'-GGGGTACCCAAGATCCACCACCCTCCAA-3' (forward) and 5'CCCAAGCTTCCGTCTCTCCCGAACAAGC-3' (reverse), and inserted into the Kpn I and Hind III sites of the luciferase reporter pGL3-Basic (Promega, Madison, WI) vector, termed pGL3-Bcl-xL-promoter. Two independent shRNAs specifically targeting lncRNA-HEIH were designed as previously described [13], and then synthesized and inserted into the SuperSilencing shRNA expression vector pGPU6/Neo (GenePharma, Shanghai, China). The target sites of lncRNAHEIH shRNAs were 5'-GCGCCUUCCCUCUAACCUUAA3' and 5'-GGCAAGAUGAACGUCUGAAAU-3'. A scrambled shRNA was used as negative control for lncRNA-HEIH shRNAs.

\section{Transfection of vectors and miR-939 inhibitors}

miR-939 inhibitors and negative control were purchased from GenePharma. The transfections of vectors and miR-939 inhibitors were performed with the Lipofectamine 3000 (Invitrogen) in accordance with the manufacturer's protocol.

\section{Construction of stable cell lines}

For the construction of lncRNA-HEIH or lncRNA-HEIHmut stably overexpressed HT-29 cells, pcDNA3.1-HEIH, pcDNA3.1-HEIH-mut, and pcDNA3.1 was transfected into HT-29 cells, and selected with neomycin $(500 \mu \mathrm{g} / \mathrm{mL})$ for four weeks. For the construction of lncRNA-HEIH stably silenced LoVo cells, the lncRNA-HEIH shRNAs and control shRNA were transfected into LoVo cells and selected with neomycin $(500 \mu \mathrm{g} / \mathrm{mL})$ for 4 weeks.

\section{Cell proliferation assay}

Cell proliferation was evaluated using the Cell Counting Kit-8 (CCK-8) assay and ethynyl deoxyuridine (EdU) incorporation assay. In brief, for CCK-8 assay, 2,000 indicated CRC cells were seeded in 96-well plates. The absorbance at $450 \mathrm{~nm}$ was detected with a CCK-8 (Dojindo Laboratories, Kumamoto, Japan) every 24 hours in accordance with the manufacturer's instructions. EdU incorporation assay was carried out with an EdU Kit (Roche) in accordance with the manufacturer's instructions.

\section{Cell apoptosis assay}

Cell apoptosis was evaluated using the TdT-mediated dUTP nick end labeling (TUNEL) assay with an In Situ Cell Death Detection Kit (Roche) in accordance with the manufacturer's instructions. The apoptotic cells were labeled with green fluorescence, analyzed by fluorescence microscopy, and counted of at least five high-power fields.

\section{In vivo tumor growth assay}

In brief, $3 \times 10^{6}$ indicted CRC cells were subcutaneously injected into 5-weeks old female athymic BALB / c nude mice (Laboratory Animal Services Centre, Southern Medical University). Subcutaneous tumor growth was detected every 7 days with a caliper for 28 days. The tumor volume was calculated using the following formula: $\mathrm{V}=0.5 \times \mathrm{a} \times \mathrm{b}^{2}(\mathrm{a}$, longitudinal diameter; $b$, latitudinal diameter). The nude mice experiments were performed in accordance with the guidelines for experimental animals of Zhujiang Hospital, Southern Medical University.

\section{Immunohistochemistry and TUNEL staining}

For immunohistochemistry staining of Ki-67, paraffin embedded tissue sections were deparaffinized, rehydrated, followed by antigen retrieval. After Ki-67 specific primary antibody (Cell Signaling Technology, Boston, MA) and a horseradish peroxidase-conjugated secondary antibody (Invitrogen) incubation, the proteins in situ were visualized with 3,3'-diaminobenzidine. For TUNEL staining, paraffin embedded tissue sections were dewaxed, rehydrated, protease treated, and permeabilized. Then TUNEL staining was performed using an In Situ Cell Death Detection Kit (Roche).

\section{RNA pull-down assay}

lncRNA-HEIH and miR-939 binding sites mutated lncRNA-HEIH (lncRNA-HEIH-mut) were in vitro transcribed from pSPT19-HEIH, and pSPT19-HEIH-mut, respec- 
tively, using the SP6 RNA polymerase (Roche) and biotinlabeled with the Biotin RNA Labeling Mix (Roche) in accordance with the manufacturer's protocols. After being treated with RNase-free DNase I (Roche), the in vitro transcribed biotinylated RNA was purified using an RNeasy Mini Kit (Qiagen, Valencia, CA). Then $3 \mu \mathrm{g}$ of purified biotinylated RNA was incubated with $1 \mathrm{mg}$ of whole-cell lysates from HT-29 cells for 1 hour at $25^{\circ} \mathrm{C}$, followed by being retrieved using the streptavidin agarose beads (Invitrogen). The RNA present in the pull-down material was quantified by qRTPCR as previously described.

\section{RNA immunoprecipitation assay}

pcDNA3.1, pcDNA3.1-HEIH, or pcDNA3.1-HEIH-mut was transfected into HT-29 cells. IncRNA-HEIH shRNA or control shRNA was co-transfected with miR-939 inhibitors or negative control into LoVo cells. 48 hours after transfection, the cells were used to undergo RNA immunoprecipitation assay (RIP) assay with a NF- $\mathrm{BB}$ specific antibody $(5 \mu \mathrm{g}$ per reaction; Millipore, Bedford, MA) and the Magna RIP RNA-Binding Protein Immunoprecipitation Kit (Millipore) in accordance with the manufacturer's protocol. The retrieved RNA was quantified by qRT-PCR as previously described.

\section{Chromatin immunoprecipitation assay}

pcDNA3.1, pcDNA3.1-HEIH, or pcDNA3.1-HEIH-mut was transfected into HT-29 cells. IncRNA-HEIH shRNA or control shRNA was co-transfected with miR-939 inhibitors or negative control into LoVo cells. Forty-eight hours after transfection, chromatin immunoprecipitation (ChIP) assay was carried out using a NF- $\mathrm{BB}$ specific antibody (5 $\mu \mathrm{g}$ per reaction, Millipore) and the EZ-Magna ChIP A/G Kit (Millipore) in accordance with the manufacturer's protocol. The retrieved DNA was quantified by qRT-PCR as previously described with the primers 5'-CTCCTGTAAGCGAGTCTG3' (forward) and 5'-TCGTTGAGCTATTGTGATG-3' (reverse), which spanning the $\mathrm{NF}-\kappa \mathrm{B}$ binding sites in $\mathrm{Bcl}-\mathrm{xL}$ promoter region.

\section{Luciferase reporter assay}

pcDNA3.1, pcDNA3.1-HEIH, or pcDNA3.1-HEIH-mut was co-transfected with pGL3-Bcl-xL-promoter and pRL-TK (Renilla luciferase expression vector, Promega) into HT-29 cells. IncRNA-HEIH shRNA or control shRNA was co-transfected with miR-939 inhibitors or negative control, and pGL3-Bcl-xL-promoter and pRL-TK into LoVo cells. Fortyeight hours after transfection, luciferase activities were measured using Dual-Luciferase Reporter Assay System (Promega) in accordance with the manufacturer's protocol. The relative firefly luciferase activity was normalized to Renilla luciferase activity.

\section{Western blot}

pcDNA3.1, pcDNA3.1-HEIH, or pcDNA3.1-HEIH-mut was transfected into HT-29 cells. IncRNA-HEIH shRNA or control shRNA was co-transfected with miR-939 inhibitors or negative control into LoVo cells. Forty-eight hours after transfection, the proteins were retrieved from these cells using RIPA buffer. Equal amounts of proteins were separated by $12 \%$ sodium dodecyl sulfate-polyacrylamide gel electrophoresis, transferred to nitrocellulose membrane, followed by being blocked with $5 \%$ bovine serum albumin. Next, the membranes were incubated with primary antibodies specific for Bcl-xL (Abcam, Hong Kong, China) or GAPDH (Cell Signaling Technology), and IRDye 800CW goat anti-rabbit IgG or IRDye 700CW goat anti-mouse IgG (Li-Cor Biosciences, Lincoln, NE), and being detected with an Odyssey infrared scanner (Li-Cor Biosciences).

\section{Statistical analysis}

Results were analyzed using the GraphPad Prism Software (San Diego, CA). Statistical comparisons were subjected to Wilcoxon signed-rank test, Pearson chi-square test, log-rank test, Student's t test, Mann-Whitney U test, or Pearson correlation analysis as indicated. $\mathrm{p}<0.05$ was considered as statistically significant.

\section{Ethical statement}

This study was conducted according to the principles expressed in the Declaration of Helsinki. This study was approved by the Ethics Committee of Zhujiang Hospital, Southern Medical University (2015-PTWK-006). All tissues were obtained with informed consent.

\section{Results}

\section{IncRNA-HEIH is increased in CRC tissues and cell lines, and correlated with poor outcome of CRC patients}

The expression of lncRNA-HEIH in 84 paired CRC and adjacent normal mucosa was detected using qRT-PCR. The result demonstrated that lncRNA-HEIH is significantly increased in CRC tissues compared with adjacent normal mucosa (Fig. 1A). Then, the correlation between lncRNA- 
A
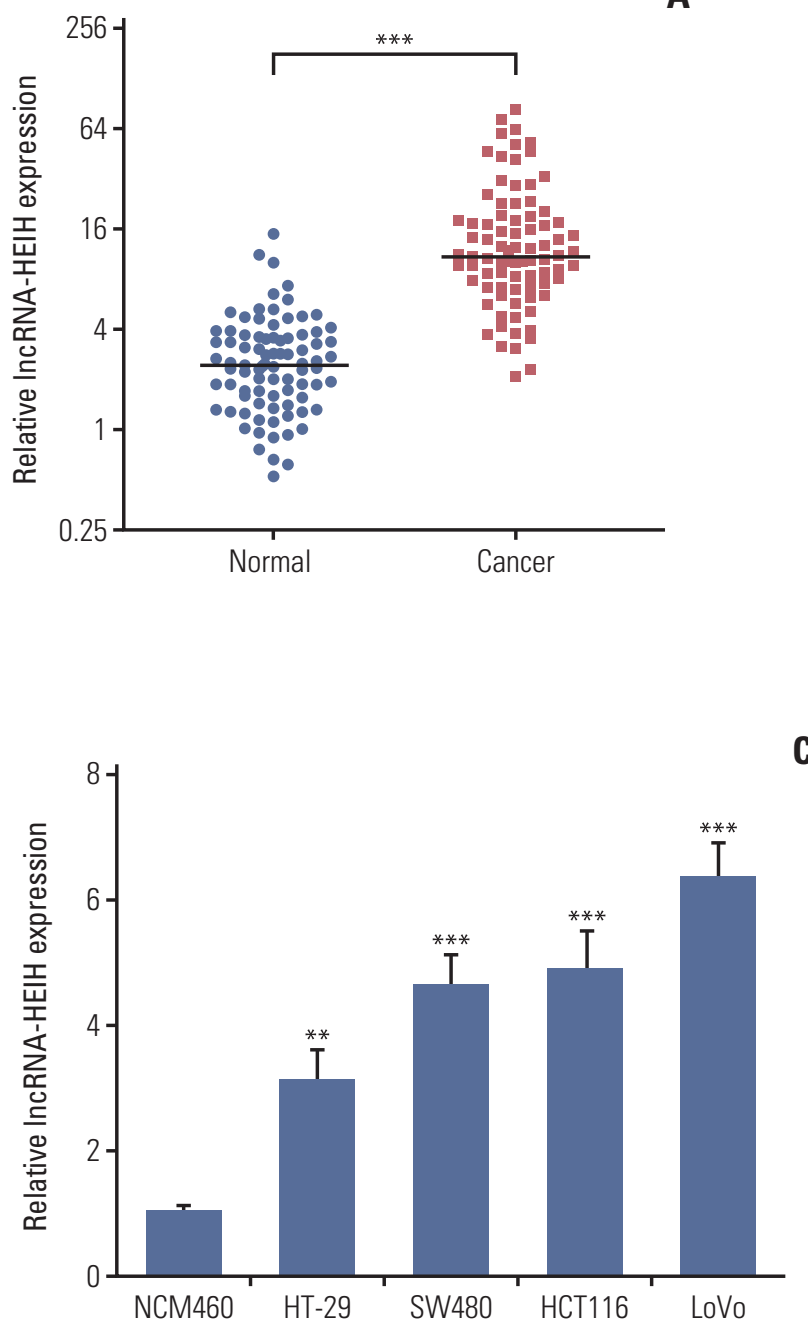

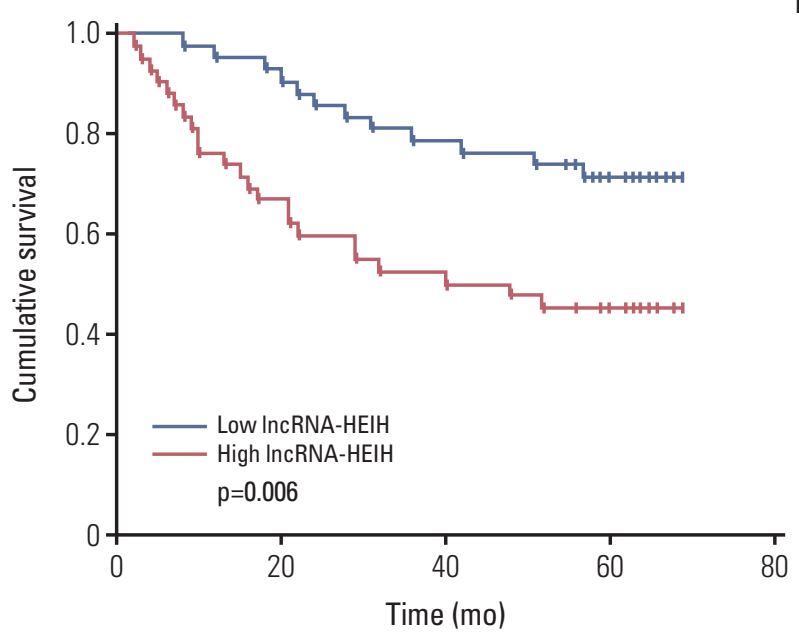

Fig. 1. Long noncoding RNA HEIH (lncRNA-HEIH) is up-regulated in colorectal cancer (CRC) and indicts poor prognosis of CRC patients. (A) The expression of lncRNA-HEIH in 84 paired CRC and adjacent normal mucosa was detected by quantitative real-time polymerase chain reaction (qRT-PCR) and normalized to glyceraldehyde 3-phosphate dehydrogenase (GAPDH). ${ }^{* * *} \mathrm{p}<0.001$ by Wilcoxon signed-rank test. (B) The 84 CRC patients were classified into two groups in accordance with the median expression value of lncRNA-HEIH in CRC tissues. Kaplan-Meier survival analysis was performed to detect the association between lncRNA-HEIH expression level and overall survival. $\mathrm{p}=0.006$ by log-rank test. (C) The expression of lncRNAHEIH in human colon normal epithelial cell line NCM460 and CRC cell lines HT-29, SW480, HCT116, and LoVo was detected by qRT-PCR and normalized to GAPDH. Results are shown as mean \pm standard deviation from three independent experiments. ${ }^{* *} \mathrm{p}<0.01,{ }^{* * *} \mathrm{p}<0.001$ by Student's $\mathrm{t}$ test.

HEIH expression and clinicopathological characteristics was analyzed in these CRC tissues. The results demonstrated that high expression of IncRNA-HEIH is associated with tumor size and invasion depth (T classification) (Table 1). A KaplanMeier survival analysis demonstrated that high expression of IncRNA-HEIH is correlated with poor overall survival (Fig. 1B). Multivariate Cox proportional hazards regression analysis further revealed that high expression of lncRNAHEIH in CRC tissues was an independent prognostic factor for reduced overall survival (Table 2). Furthermore, the expression of IncRNA-HEIH in human colon normal epithelial cell line NCM460 and CRC cell lines HT-29, SW480, HCT116, and LoVo was also detected using qRT-PCR. The result demonstrated that lncRNA-HEIH is increased in CRC 
Table 1. Correlation between lncRNA-HEIH expression level and clinicopathological features in CRC patients

\begin{tabular}{|c|c|c|c|}
\hline \multirow{2}{*}{ Characteristic } & \multicolumn{2}{|c|}{ lncRNA-HEIH expression } & \multirow{2}{*}{ p-value } \\
\hline & Low $(n=42)$ & High $(n=42)$ & \\
\hline \multicolumn{4}{|l|}{ Sex } \\
\hline Male & $26(31.0)$ & $23(27.4)$ & 0.507 \\
\hline Female & $16(19.0)$ & $19(22.6)$ & \\
\hline \multicolumn{4}{|l|}{ Age (yr) } \\
\hline$<50$ & $8(9.5)$ & $6(7.1)$ & 0.558 \\
\hline$\geq 50$ & $34(40.5)$ & $36(42.9)$ & \\
\hline \multicolumn{4}{|l|}{ Tumor site } \\
\hline Right colon & $12(14.3)$ & $14(16.7)$ & 0.801 \\
\hline Left colon & $10(11.9)$ & $11(13.1)$ & \\
\hline Rectum & $20(23.8)$ & $17(20.2)$ & \\
\hline \multicolumn{4}{|c|}{ Tumor size (diameter in $\mathrm{cm}$ ) } \\
\hline$<5$ & $27(32.1)$ & $16(19.0)$ & $0.016^{*}$ \\
\hline$\geq 5$ & $15(17.9)$ & $26(31.0)$ & \\
\hline \multicolumn{4}{|l|}{ Differentiation } \\
\hline Good/Moderate & $33(39.3)$ & $28(33.3)$ & 0.221 \\
\hline Poor & $9(10.7)$ & $14(16.7)$ & \\
\hline \multicolumn{4}{|l|}{ T classification } \\
\hline $\mathrm{T} 1+\mathrm{T} 2$ & $24(28.6)$ & $14(16.7)$ & $0.028^{*}$ \\
\hline $\mathrm{T} 3+\mathrm{T} 4$ & $18(21.4)$ & $28(33.3)$ & \\
\hline \multicolumn{4}{|l|}{$\mathrm{N}$ classification } \\
\hline N0 & $33(39.3)$ & $25(29.8)$ & 0.059 \\
\hline $\mathrm{N} 1+\mathrm{N} 2$ & $9(10.7)$ & $17(20.2)$ & \\
\hline
\end{tabular}

p-value was acquired by Pearson chi-square test. IncRNA-HEIH, long noncoding RNA HEIH; CRC, colorectal cancer. *Statistically significant $(\mathrm{p}<0.05)$.

Table 2. Multivariate analysis of several variables for RFS and OS

\begin{tabular}{|c|c|c|c|c|}
\hline \multirow{2}{*}{ Variable } & \multicolumn{2}{|c|}{ Univariate Cox's regression analysis } & \multicolumn{2}{|c|}{ Multivariate Cox's regression analysis } \\
\hline & Hazard ratio $(95 \% \mathrm{CI})$ & p-value & Hazard ratio $(95 \% \mathrm{CI})$ & p-value \\
\hline lncRNA-HEIH (high vs. low) & $2.550(1.302-4.995)$ & 0.006 & $1.456(1.017-2.084)$ & 0.040 \\
\hline Sex (male vs. female) & $1.020(0.727-1.430)$ & 0.909 & - & - \\
\hline Age $(<50$ yr vs. $\geq 50$ yr $)$ & $0.774(0.460-1.304)$ & 0.336 & - & - \\
\hline Tumor site (colon vs. rectum) & $1.176(0.835-1.657)$ & 0.354 & - & - \\
\hline Tumor size $(\geq 5 \mathrm{~cm}$ vs. $<5 \mathrm{~cm})$ & $1.819(0.930-3.557)$ & 0.080 & - & - \\
\hline Differentiation (poor vs. good/moderate) & $1.466(1.045-2.057)$ & 0.027 & $1.470(1.045-2.069)$ & 0.027 \\
\hline T classification (T3+T4 vs. T1+T2) & $1.425(1.004-2.020)$ & 0.047 & - & - \\
\hline $\mathrm{N}$ classification (N1+N2 vs. N0) & $1.551(1.110-2.166)$ & 0.010 & $1.465(1.040-2.065)$ & 0.029 \\
\hline
\end{tabular}

p-value was acquired by Cox proportional hazards regression. RFS, recurrence-free survival; OS, overall survival; CI, confidence interval; lncRNA-HEIH, long noncoding RNA HEIH. 
A
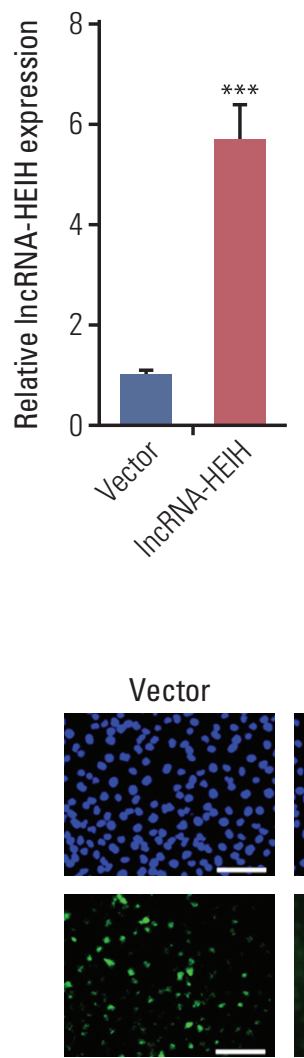

InCRNA-HEIH

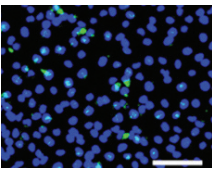

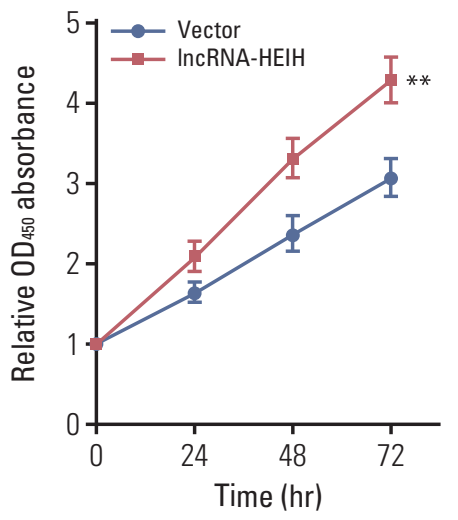

D
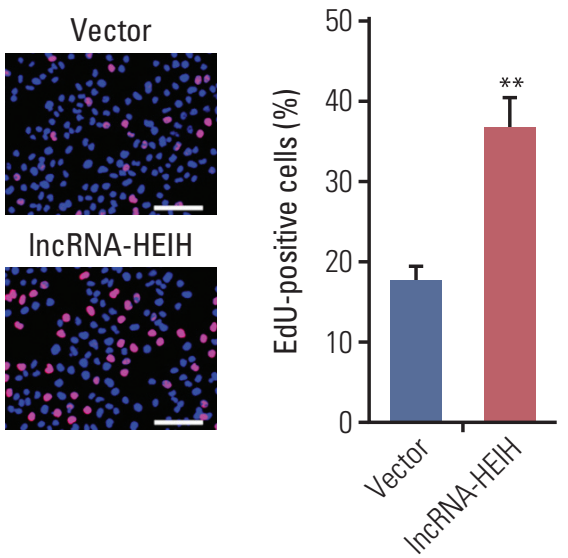

C

$\mathbf{E}$

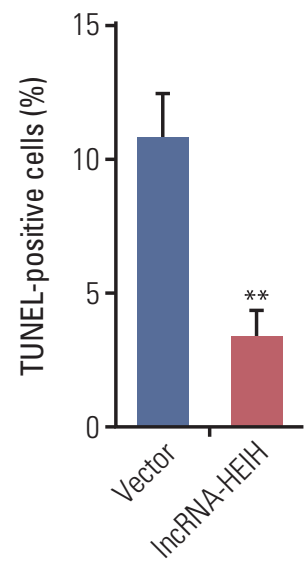

Fig. 2. Enhanced expression of long noncoding RNA HEIH (lncRNA-HEIH) promotes colorectal cancer tumorigenesis. (A) The expression of IncRNA-HEIH in lncRNA-HEIH stably overexpressed and control HT-29 cells was detected by quantitative real-time polymerase chain reaction and normalized to glyceraldehyde 3-phosphate dehydrogenase. (B) Cell proliferation rate of lncRNA-HEIH stably overexpressed and control HT-29 cells were detected by the Cell Counting Kit- 8 assays. (C) Proliferative cells of lncRNA-HEIH stably overexpressed and control HT-29 were labeled with ethynyl deoxyuridine (EdU). Red color indicts EdU-positive cells. Scale bars $=100 \mu \mathrm{m}$. (D) The level of apoptosis in IncRNA-HEIH stably overexpressed and control HT-29 cells was detected by TdT-mediated dUTP nick end labeling (TUNEL) staining. Blue color indicts TUNELpositive cells. Scale bars $=100 \mu \mathrm{m}$. For A-D, results are shown as mean \pm standard deviation (SD). from three independent experiments. ${ }^{* *} \mathrm{p}<0.01,{ }^{* * *} \mathrm{p}<0.001$ by Student's $\mathrm{t}$ test. (E, F) lncRNA-HEIH stably overexpressed and control HT-29 cells were subcutaneously injected into nude mice. Tumor volumes were detected every 7 days (E). (Continued to the next page)

cell lines compared with human colon normal epithelial cell line (Fig. 1C). Taken together, these results demonstrated that IncRNA-HEIH is increased in CRC tissues and cell lines, and correlated with tumor size, invasion depth, and poor prognosis of CRC patients.

\section{Enhanced expression of IncRNA-HEIH promotes CRC growth in vitro and in vivo}

To determine the effects of IncRNA-HEIH on CRC cellular behavior, we transfected IncRNA-HEIH expression plasmid pcDNA3.1-HEIH into HT-29 cells and acquired lncRNAHEIH stably overexpressed HT-29 cells (Fig. 2A). The CCK- 
$\mathbf{F}$

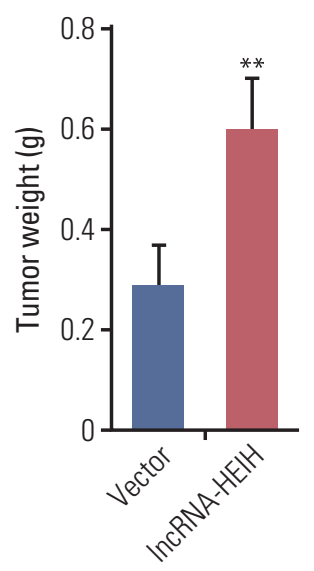

G

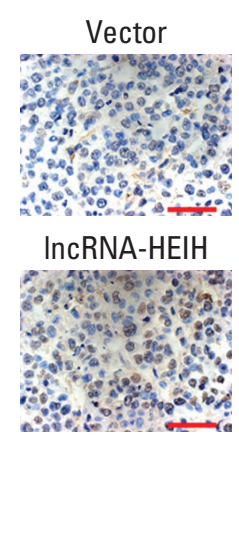

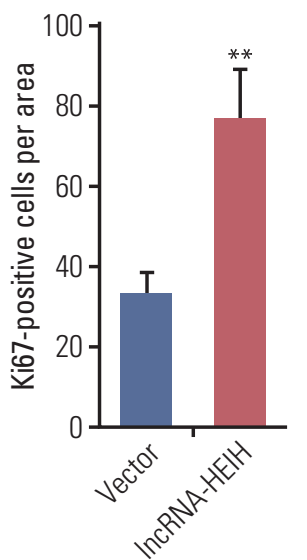

Fig. 2. (Continued from the previous page) Tumor weights were detected at the 28th day after injection (F). (G) Tumors generated from subcutaneous injection with lncRNA-HEIH stably overexpressed and control HT-29 cells were immunohistochemistry stained for Ki-67. Scale bars $=50 \mu \mathrm{m}$. (H) The same tumors as in panel G were stained for TUNEL. Scale bars=100 $\mu \mathrm{m}$. For E-H, results are shown as mean \pm SD from six mice. ** $\mathrm{p}<0.01$ by Mann-Whitney $U$ test.

8 assays demonstrated that enhanced expression of lncRNAHEIH significantly increases the rate of cell proliferation in HT-29 cells (Fig. 2B). The EdU incorporation assays also demonstrated that overexpression of lncRNA-HEIH promotes HT-29 cell proliferation (Fig. 2C). The TUNEL staining demonstrated that enhanced expression of lncRNA-HEIH decreases the percentage of cells undergoing apoptosis in HT-29 cells (Fig. 2D). The effects of lncRNA-HEIH on CRC cellular behavior in vivo were also investigated. IncRNAHEIH stably overexpressed and control HT-29 cells were subcutaneously injected into nude mice. The subcutaneous tumor model demonstrated that tumors grew faster in HT-29/1ncRNA-HEIH overexpression group compared with HT-29/ control group (Fig. 2E and F). Immunohistochemistry staining of proliferation marker $\mathrm{Ki}-67$ demonstrated that enhanced expression of lncRNA-HEIH increases the number of cells undergoing proliferation in tumors formed from HT-29 cells (Fig. 2G). The TUNEL staining of subcutaneous tumors demonstrated that enhanced expression of lncRNAHEIH decreases the number of cells undergoing apoptosis (Fig. 2H). Taken together, these results demonstrated that enhanced expression of lncRNA-HEIH promotes CRC growth in vitro and in vivo.

\section{Knockdown of lncRNA-HEIH inhibits CRC growth in vitro and in vivo}

To completely investigate the effects of lncRNA-HEIH on CRC cellular behavior, we transfected two independent lncRNA-HEIH shRNAs into LoVo cells and acquired lnc-
RNA-HEIH stably knocked-down LoVo cells (Fig. 3A). The CCK-8 assays demonstrated that knockdown of 1ncRNAHEIH by both shRNAs decreases the rate of cell proliferation in LoVo cells (Fig. 3B). The EdU incorporation assays also demonstrated that knockdown of lncRNA-HEIH by both shRNAs inhibits LoVo cell proliferation (Fig. 3C). The TUNEL staining demonstrated that knockdown of lncRNA-HEIH increases the percentage of cells undergoing apoptosis in LoVo cells (Fig. 3D). The effects of lncRNA-HEIH knockdown on CRC cellular behavior in vivo were further investigated. IncRNA-HEIH stably silenced and control LoVo cells were subcutaneously injected into nude mice. The subcutaneous tumor model demonstrated that tumors grew more slowly in LoVo/lncRNA-HEIH knockdown group compared with LoVo/ control group (Fig. 3E and F). Immunohistochemistry staining of Ki-67 demonstrated that knockdown of lncRNA-HEIH decreases the number of cells undergoing proliferation in tumors formed from LoVo cells (Fig. 3G). The TUNEL staining of subcutaneous tumors demonstrated that knockdown of lncRNA-HEIH increases the number of cells undergoing apoptosis (Fig. $3 \mathrm{H}$ ). Taken together, these results demonstrated that knockdown of lncRNA-HEIH inhibits CRC growth in vitro and in vivo.

\section{IncRNA-HEIH physically associates with miR-939 and suppresses the binding between miR-939 and NF-кB}

To explore the molecular mechanisms underlying the effects of lncRNA-HEIH on CRC cellular behavior, we performed bioinformatics analysis by TargetScan and found 
A

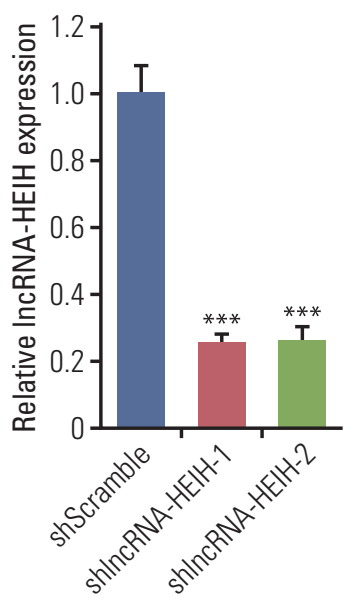

B

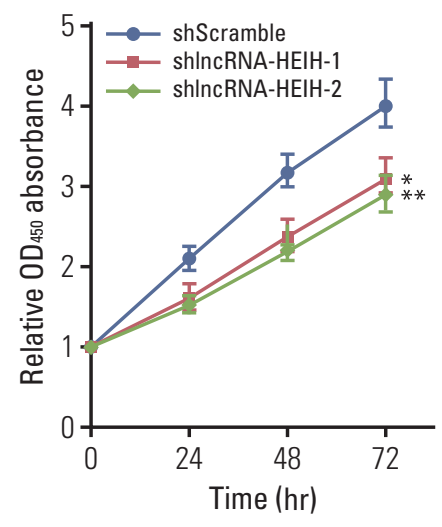

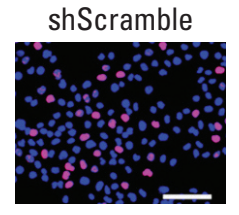

shlncRNA-HEIH-1

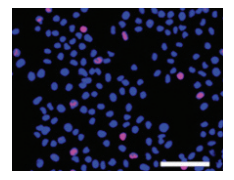

shlncRNA-HEIH-2

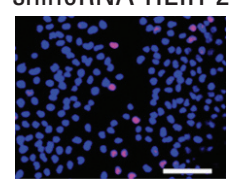

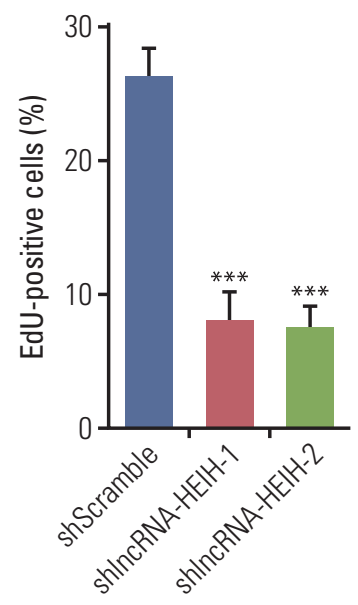

D

E
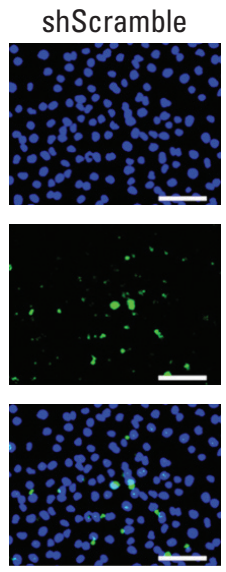
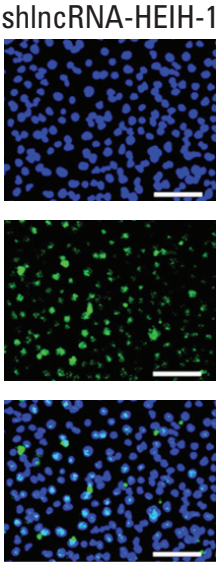
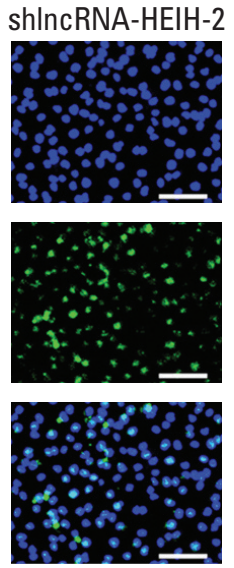
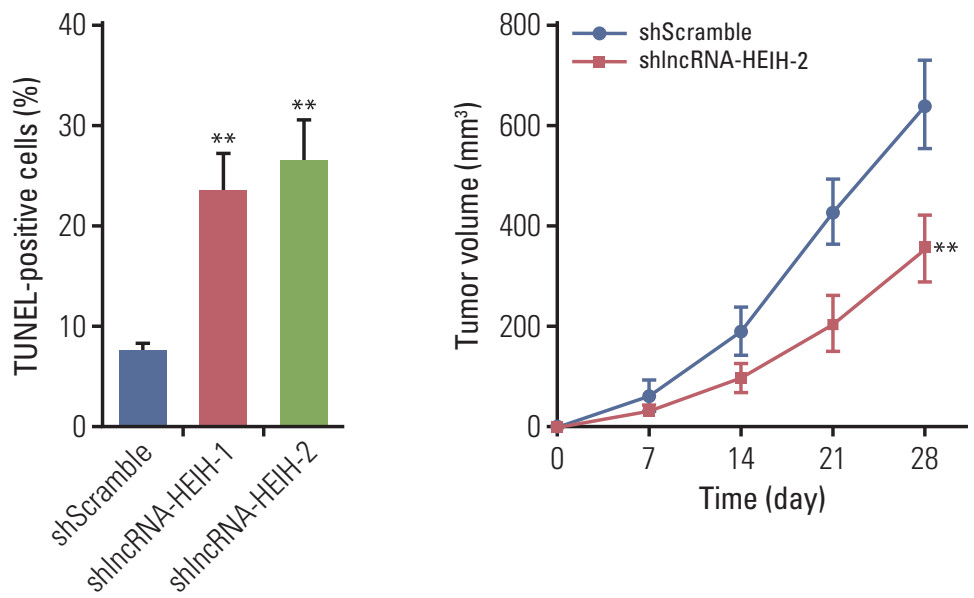

Fig. 3. Knockdown of long noncoding RNA HEIH (lncRNA-HEIH) inhibits colorectal cancer tumorigenesis. (A) The expression of lncRNA-HEIH in lncRNA-HEIH stably knocked-down and control LoVo cells was detected by quantitative real-time polymerase chain reaction and normalized to glyceraldehyde 3-phosphate dehydrogenase. (B) Cell proliferation rate of IncRNA-HEIH stably knocked-down and control LoVo cells were detected by the Cell Counting Kit-8 assays. (C) Proliferative cells of lncRNA-HEIH stably knocked-down and control LoVo were labeled with ethynyl deoxyuridine (EdU). Red color indicts EdU-positive cells. Scale bars $=100 \mu \mathrm{m}$. (D) The level of apoptosis in lncRNA-HEIH stably knocked-down and control LoVo cells was detected by TdT-mediated dUTP nick end labeling (TUNEL) staining. Blue color indicts TUNEL-positive cells. Scale bars $=100 \mu \mathrm{m}$. For A-D, results are shown as mean \pm standard deviation (SD) from three independent experiments. ${ }^{*} \mathrm{p}<0.05,{ }^{* *} \mathrm{p}<0.01,{ }^{* * *} \mathrm{p}<0.001$ by Student's $\mathrm{t}$ test. (E, F) IncRNA-HEIH stably knocked-down and control LoVo cells were subcutaneously injected into nude mice. Tumor volumes were detected every 7 days (E). (Continued to the next page)

three putative miR-939 response elements on lncRNA-HEIH (Fig. 4A). To investigate whether lncRNA-HEIH could physically associates with miR-939, we carried out RNA pulldown assays using in vitro transcribed biotinylated lncRNAHEIH. The RNA pull-down assays demonstrated that lnc-
RNA-HEIH specifically binds to miR-939 and the primary miR-939 (pri-miR-939), and while mutation of the miR-939 response elements on lncRNA-HEIH abolishes the binding of lncRNA-HEIH to miR-939 and pri-miR-939 (Fig. 4B). In our previous study, we found that miR-939 could physi- 
$\mathbf{F}$

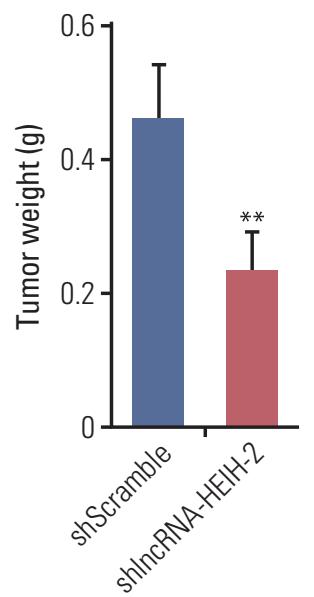

G

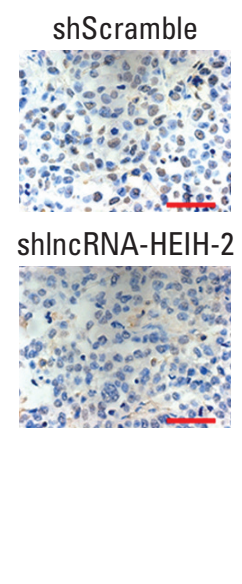

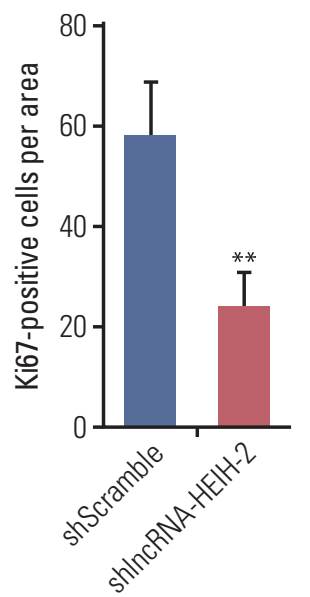

H
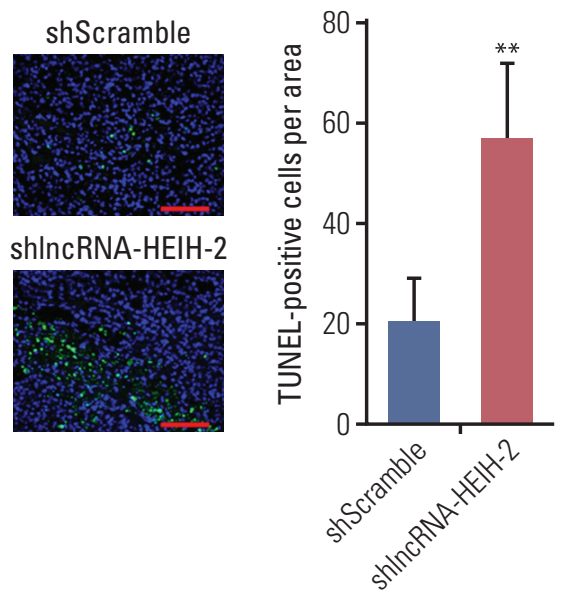

Fig. 3. (Continued from the previous page) Tumor weights were detected at the 28th day after injection (F). (G) Tumors generated from subcutaneous injection with lncRNA-HEIH stably knocked-down and control LoVo cells were immunohistochemistry stained for Ki-67. Scale bars $=50 \mu \mathrm{m}$. $(\mathrm{H})$ The same tumors as in panel $\mathrm{G}$ were stained for TUNEL. Scale bars $=100 \mu \mathrm{m}$. For E-H, results are shown as mean \pm SD from six mice. ${ }^{* *} \mathrm{p}<0.01$ by Mann-Whitney $\mathrm{U}$ test.

cally bind to transcription factor NF- $\mathrm{\kappa B}$ and produce transcriptional regulation of NF- $\mathrm{kB}$ target gene Bcl-xL. Next, we investigated whether the association between lncRNA-HEIH and miR-939 influences the binding of miR-939 to NF-кB. RIP assays using NF- $\mathrm{KB}$ specific antibody demonstrated that enhanced expression of lncRNA-HEIH significantly decreases the binding of NF- $\mathrm{kB}$ to miR-939 and pri-miR-939, and while mutation of the miR-939 response elements on lncRNA-HEIH abolishes the effects of IncRNA-HEIH on the binding of NF-kB to miR-939 and pri-miR-939 (Fig. 4C). The RIP assays also demonstrated that knockdown of lncRNA$\mathrm{HEIH}$ significantly increases the binding of NF- $\mathrm{kB}$ to miR939 and pri-miR-939, and while inhibition of miR-939 reverses the effects of lncRNA-HEIH knockdown on the binding of NF-kB to miR-939 and pri-miR-939 (Fig. 4D). Taken together, these results demonstrated that lncRNAHEIH physically associates with miR-939 and suppresses the binding between miR-939 and NF-kB.

\section{IncRNA-HEIH counteracts miR-939-mediated transcrip- tional repression of $\mathrm{Bcl}-\mathrm{xL}$}

In our previous study, we have found that through binding to NF- $\mathrm{kB}$, miR-939 decreases the occupancy of NF- $\mathrm{kB}$ on the promoter region of the $\mathrm{Bcl}-\mathrm{xL}$ gene and transcriptionally represses Bcl-xL expression [19]. Bcl-xL is a NF- $\mathrm{kB}$ target and has critical anti-apoptotic roles in many cancers [20]. Next, we investigated the effects of IncRNA-HEIH on the transcriptional regulation of Bcl-xL by miR-939. The ChIP assays using NF- $\mathrm{BB}$ specific antibody demonstrated that enhanced expression of lncRNA-HEIH significantly increases the binding of NF- $\mathrm{KB}$ to $\mathrm{Bcl}-\mathrm{xL}$ promoter region, and while mutation of the miR-939 response elements on lncRNA-HEIH abolishes the effects of lncRNA-HEIH on the binding of NF- $\kappa B$ to Bcl-xL promoter (Fig. 5A). The ChIP assays also demonstrated that knockdown of lncRNA-HEIH significantly decreases the binding of NF- $\mathrm{KB}$ to $\mathrm{Bcl}-\mathrm{xL}$ promoter region, and while inhibition of miR-939 reverses the effects of lncRNA-HEIH knockdown on the binding of NF- $\kappa B$ to Bcl$\mathrm{xL}$ promoter (Fig. 5B). These data suggested that through competitively binding to miR-939, IncRNA-HEIH promotes the binding of NF- $\mathrm{kB}$ to $\mathrm{Bcl}-\mathrm{xL}$ promoter. Then, the luciferase reporter assays were performed to investigate the effects of lncRNA-HEIH on transcription activity of Bcl-xL. Bcl-xL promoter region spanning the NF- $\mathrm{\kappa B}$ binding sites was inserted into the luciferase vector. The luciferase reporter assays demonstrated that enhanced expression of lncRNA-HEIH significantly increases the luciferase activities of the construct containing Bcl-xL promoter, and while mutation of the miR939 response elements on IncRNA-HEIH abolishes the increasing of the luciferase activities (Fig. 5C). The luciferase reporter assays also demonstrated that knockdown of lncRNA-HEIH significantly decreases the luciferase activities of the construct containing Bcl-xL promoter, and while inhibition of miR-939 reverses the decreasing of the luciferase activities (Fig. 5D). These data suggested that through competitively binding to miR-939, IncRNA-HEIH increases the transcription activity of Bcl-xL. 
A
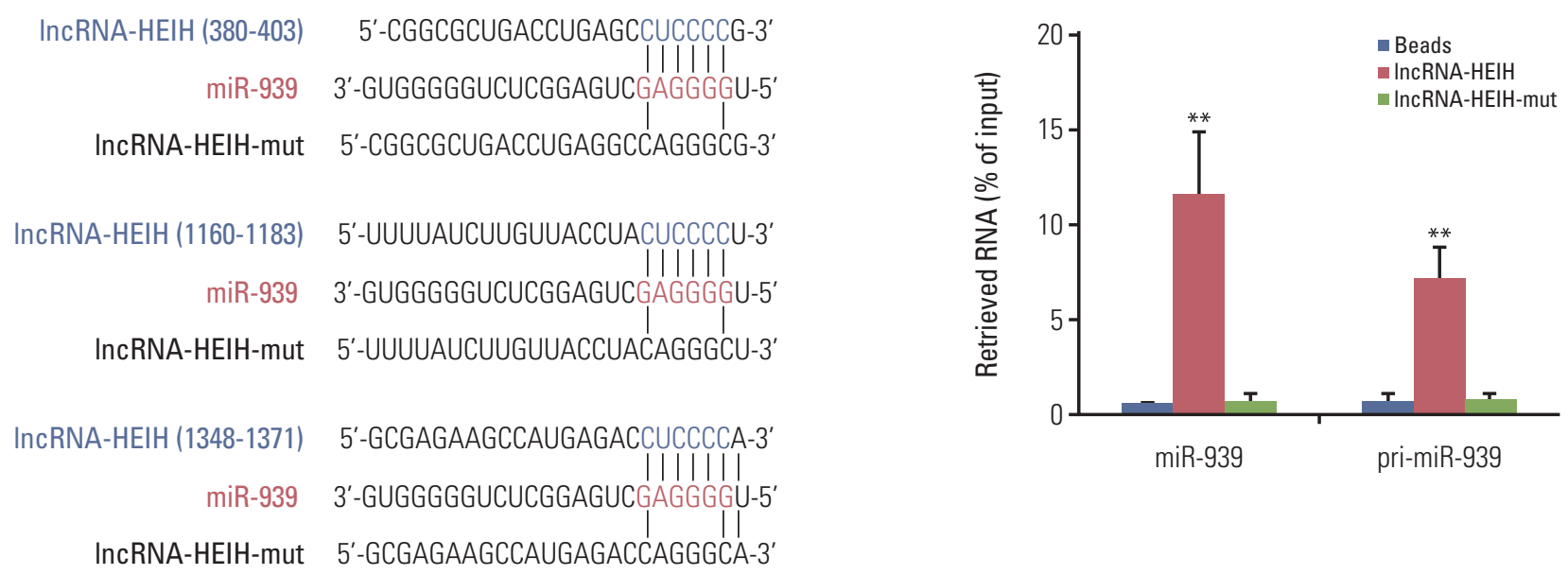

C
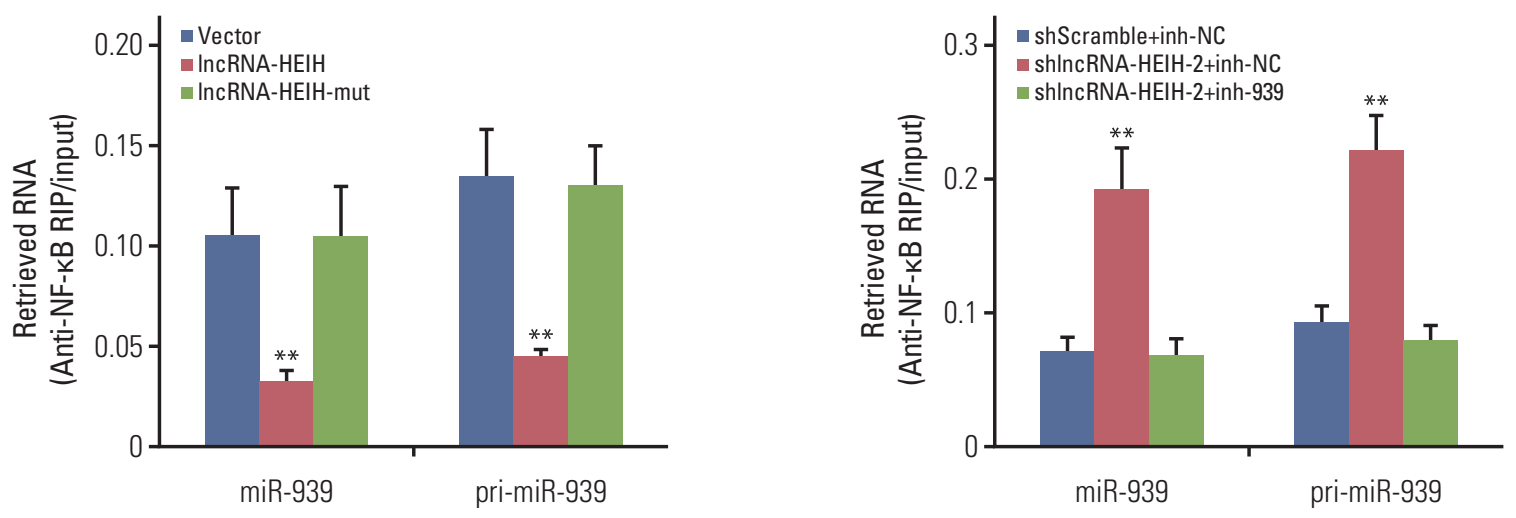

Fig. 4. Long noncoding RNA HEIH (lncRNA-HEIH) physically binds to miR-939 and counteracts the binding between miR939 and nuclear factor $\mathrm{\kappa B}$ (NF-кB). (A) Schematic outlining the predicted miR-939 binding sites on IncRNA-HEIH. The red nucleotides indict the seed sequences of miR-939. (B) HT-29 cell lysates were incubated with biotinylated lncRNA-HEIH or the miR-939 binding sites mutated lncRNA-HEIH (IncRNA-HEIH-mut); after pull-down, RNAs were retrieved and detected by quantitative real-time polymerase chain reaction (qRT-PCR), and normalized to input. (C) After transient transfection of lncRNA-HEIH or lncRNA-HEIH-mut into HT-29 cells, RNA immunoprecipitation assay (RIP) assays with NF-kB specific antibody were performed, and the retrieved RNA was detected by qRT-PCR and normalized to input. (D) After co-transfection of lncRNA-HEIH knockdown plasmid and miR-939 inhibitors into LoVo cells, RIP assays with NF-kB specific antibody were performed, and the retrieved RNA was detected by qRT-PCR and normalized to input. Results are shown as mean \pm standard deviation from three independent experiments. ${ }^{* *} \mathrm{p}<0.01$ by Student's $\mathrm{t}$ test.

The effects of lncRNA-HEIH on the expression of Bcl-xL were further detected by qRT-PCR and western blot assays. The qRT-PCR assays demonstrated that enhanced expression of lncRNA-HEIH significantly increases Bcl-xL mRNA levels, which was abolished by the mutation of the miR-939 response elements on lncRNA-HEIH (Fig. 5E). Knockdown of lncRNA-HEIH significantly decreases Bcl-xL mRNA lev- els, which was abolished by miR-939 inhibition (Fig. 5F). Western blot assays demonstrated that enhanced expression of lncRNA-HEIH significantly increases Bcl-xL protein levels, which was abolished by the mutation of the miR-939 response elements on lncRNA-HEIH (Fig. 5G). Knockdown of lncRNA-HEIH significantly decreases Bcl-xL protein levels, which was abolished by miR-939 inhibition (Fig. 5H). 


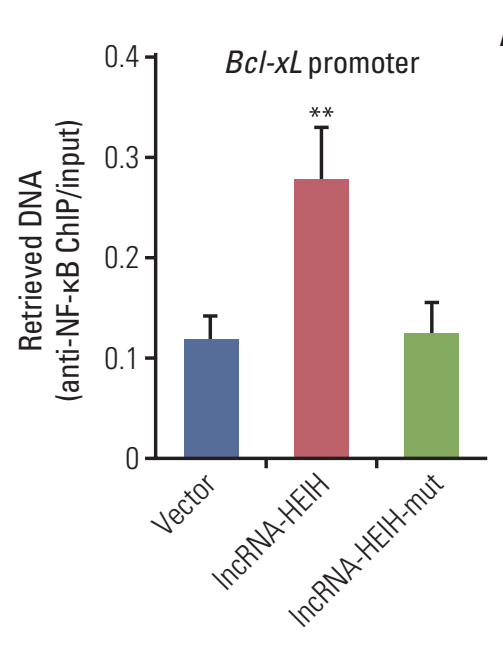

A

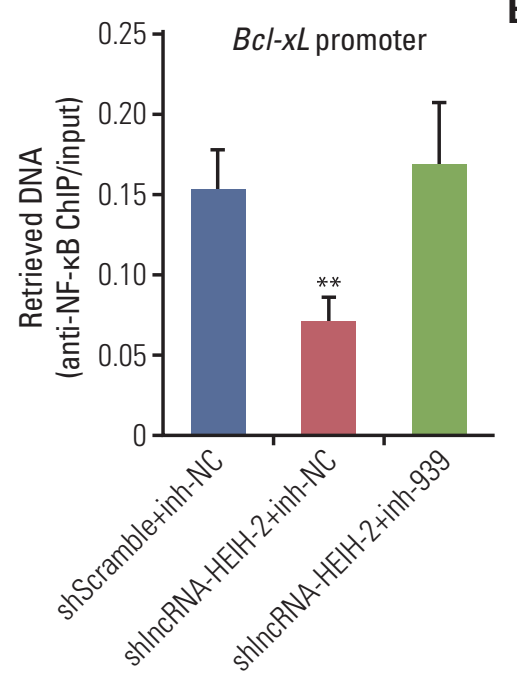

B

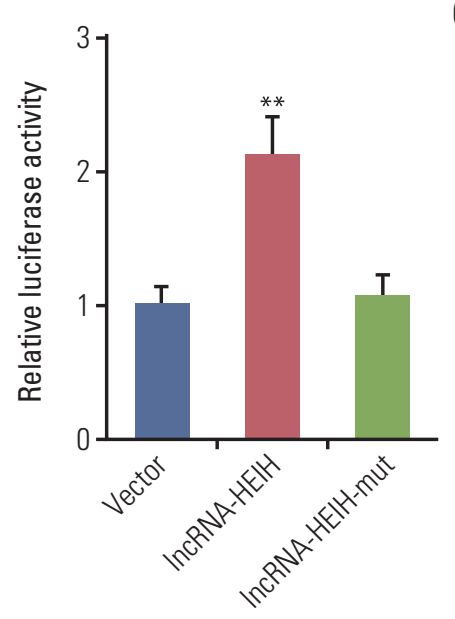

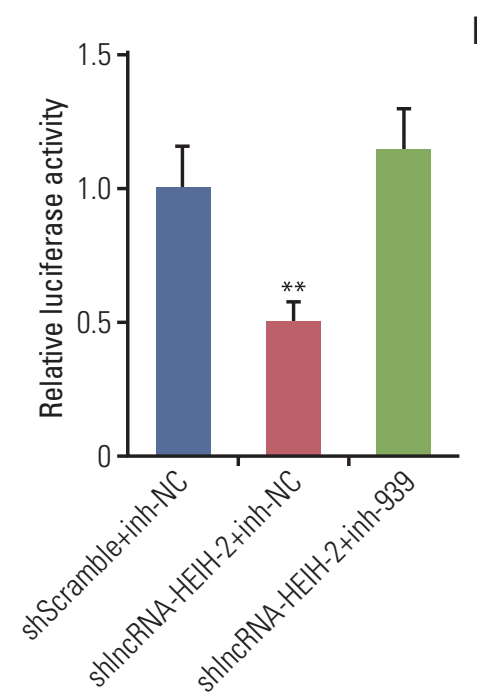

D

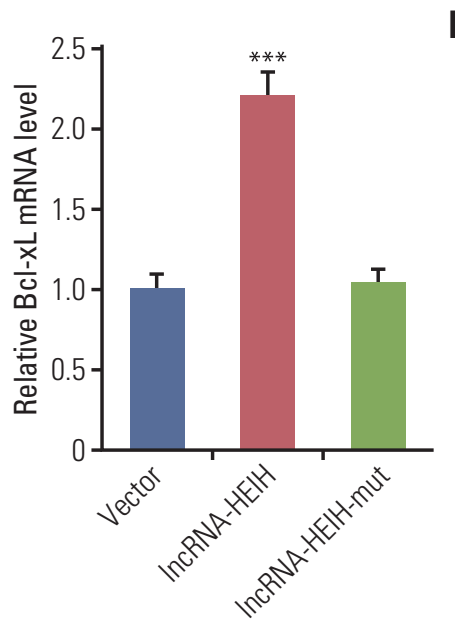

E

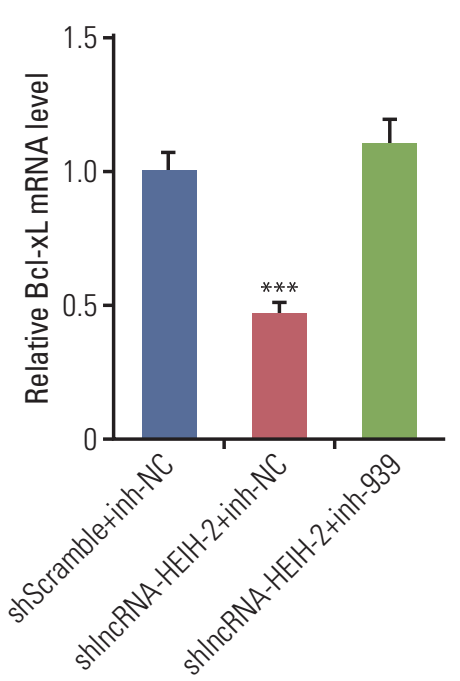

Fig. 5. Long noncoding RNA HEIH (lncRNA-HEIH) counteracts miR-939-mediated transcriptional repression of Bcl-xL. (A) After transient transfection of lncRNA-HEIH or lncRNA-HEIH-mut into HT-29 cells, chromatin immunoprecipitation (ChIP) assays with nuclear factor $\mathrm{\kappa B}(\mathrm{NF}-\mathrm{\kappa B})$ specific antibody were performed, and the retrieved DNA was detected by quantitative real-time polymerase chain reaction (qRT-PCR) and normalized to input. (B) After co-transfection of lncRNA-HEIH knockdown plasmid and miR-939 inhibitors into LoVo cells, ChIP assays with NF-kB specific antibody were performed, and the retrieved DNA was detected by qRT-PCR and normalized to input. (C) After co-transfection of lncRNA-HEIH or lncRNAHEIH-mut and luciferase reporter containing Bcl-xL promoter into HT-29 cells, the luciferase activities were measured. Results are shown as the relative ratio of firefly luciferase activity to Renilla luciferase activity. (D) After co-transfection of lncRNAHEIH knockdown plasmid, miR-939 inhibitors, and luciferase reporter containing Bcl-xL promoter into LoVo cells, the luciferase activities were measured. Results are shown as in panel C. (E) After transient transfection of lncRNA-HEIH or lncRNA-HEIH-mut into HT-29 cells, Bcl-xL mRNA levels were detected by qRT-PCR and normalized to glyceraldehyde 3-phosphate dehydrogenase (GAPDH). (F) After co-transfection of lncRNA-HEIH knockdown plasmid and miR-939 inhibitors into LoVo cells, Bcl-xL mRNA levels were detected by qRT-PCR and normalized to GAPDH. (Continued to the next page) 
G
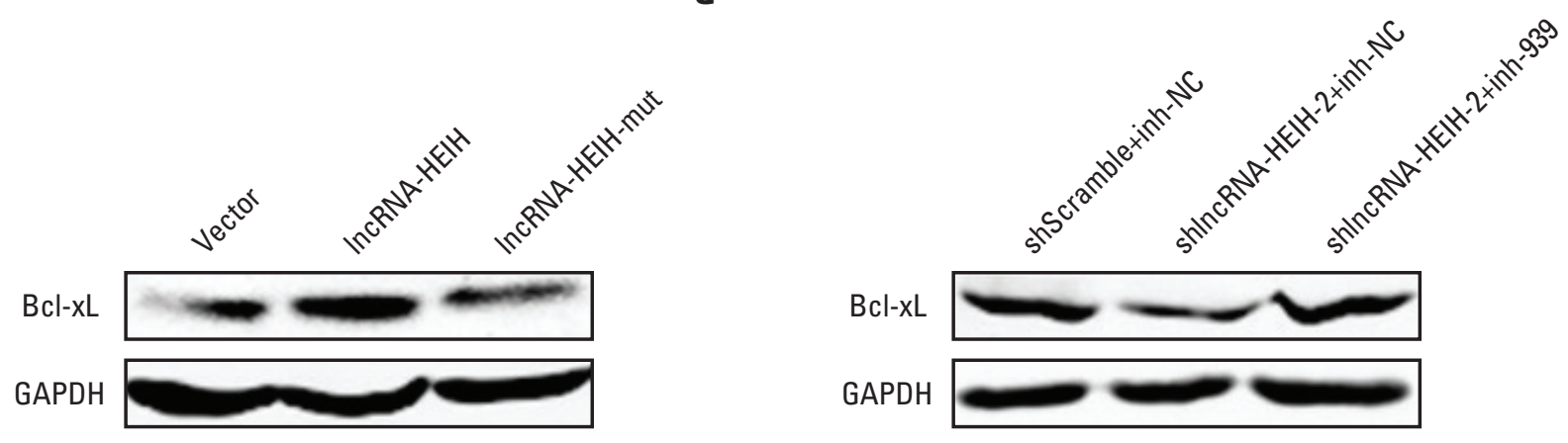

Fig. 5. (Continued from the previous page) (G) After transient transfection of lncRNA-HEIH or lncRNA-HEIH-mut into HT-29 cells, Bcl-xL protein levels were detected by western blot and normalized to GAPDH. (H) After co-transfection of lncRNA-HEIH knockdown plasmid and miR-939 inhibitors into LoVo cells, Bcl-xL protein levels were detected by western blot and normalized to GAPDH. Results are shown as mean \pm standard deviation from three independent experiments. ${ }^{* *} \mathrm{p}<0.01,{ }^{* * *} \mathrm{p}<0.001$ by Student's t test.

Taken together, these results demonstrated that lncRNA$\mathrm{HEIH}$ increases the transcription activity and expression of Bcl-xL via competitively binding to miR-939 and suppressing the binding between miR-939 and NF-кB.

\section{Bcl-xL expression is positively correlated with lncRNA- HEIH expression in CRC tissues}

The expression of $\mathrm{Bcl}-\mathrm{xL}$ in the same 84 paired CRC and adjacent normal mucosa was detected using qRT-PCR to determine whether $\mathrm{Bcl}-\mathrm{xL}$ expression is associated with lncRNA-HEIH expression in clinical tissue samples. The result demonstrated that $\mathrm{Bcl}-\mathrm{xL}$ is also significantly increased in CRC tissues compared with adjacent normal mucosa (Fig. 6A). A correlation analysis demonstrated that Bcl-xL expression level is positively correlated with that of lncRNAHEIH in CRC tissues (Fig. 6B).

\section{The effects of lncRNA-HEIH on CRC tumorigenesis are dependent on the binding to miR-939}

To determine whether the effects of lncRNA-HEIH on CRC tumorigenesis are dependent on the competitive binding to miR-939, we transfected miR-939 response elements mutated lncRNA-HEIH expression plasmid into HT-29 cells and acquired lncRNA-HEIH-mut stably overexpressed HT-29 cells with the same overexpression efficiency as IncRNA-HEIH stably overexpressed HT-29 cells (Fig. 7A). The CCK-8 assays demonstrated that the mutation of the miR-939 response elements on lncRNA-HEIH abolishes the increasing of the cell proliferation rate caused by lncRNAHEIH overexpression in HT-29 cells (Fig. 7B). The EdU incorporation assays also demonstrated that the mutation of the miR-939 response elements on lncRNA-HEIH abolishes the pro-proliferative effects of lncRNA-HEIH in HT-29 cells (Fig. 7C). The TUNEL staining demonstrated that the mutation of the miR-939 response elements on lncRNA-HEIH abolishes the decreasing of the percentage of cells undergoing apoptosis caused by lncRNA-HEIH overexpression in HT-29 cells (Fig. 7D). The subcutaneous tumor model demonstrated that the mutation of the miR-939 response elements on lncRNA-HEIH abolishes the pro-growth effects of lncRNA-HEIH (Fig. 7E and F). Immunohistochemistry staining of proliferation marker Ki-67 demonstrated that the mutation of the miR-939 response elements on lncRNA$\mathrm{HEIH}$ abolishes the increasing of the number of cells undergoing proliferation in tumors formed from HT-29 cells caused by lncRNA-HEIH overexpression (Fig. 7G). The TUNEL staining of subcutaneous tumors demonstrated that the mutation of the miR-939 response elements on lncRNAHEIH abolishes the decreasing of the number of cells undergoing apoptosis caused by lncRNA-HEIH overexpression (Fig. 7H). Taken together, these results demonstrated that the effects of lncRNA-HEIH on CRC tumorigenesis are dependent on the competitively binding to miR-939.

\section{Discussion}

CRC is the third most common and one of the most frequent cause of cancer-related death worldwide [1]. Increased cell survival capability is critical during CRC tumorigenesis 

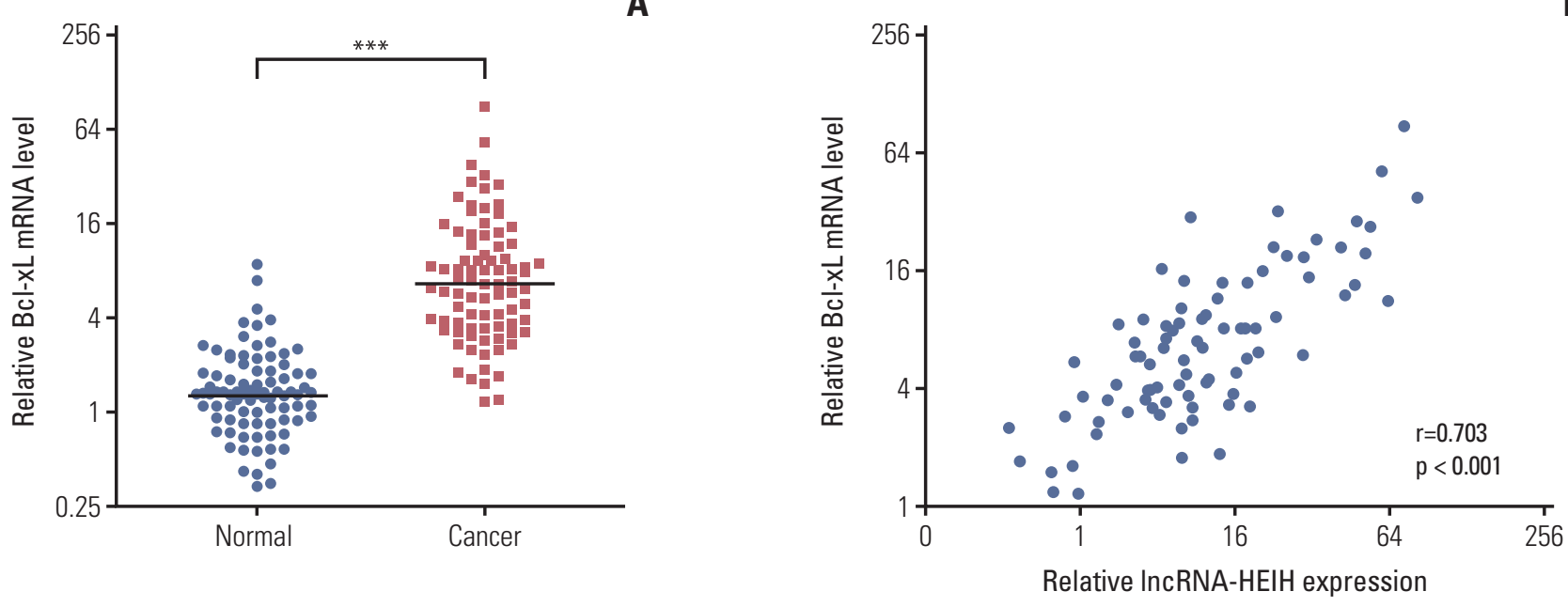

Fig. 6. Bcl-xL mRNA level is positively correlated with long noncoding RNA HEIH (lncRNA-HEIH) expression level in colorectal cancer (CRC) tissues. (A) Bcl-xL mRNA levels in 84 paired CRC and adjacent normal mucosa were detected by quantitative real-time polymerase chain reaction and normalized to glyceraldehyde 3-phosphate dehydrogenase. ${ }^{* * *} \mathrm{p}<0.001$ by Wilcoxon signed-rank test. (B) The correlation between lncRNA-HEIH and Bcl-xL expression levels in 84 CRC tissues was detected by Pearson correlation analysis.

and progression [21]. Previously, people mainly focused their attentions on proteins with oncogenic or tumor-suppressing roles, such as KRAS, p53, and adenomatous polyposis coli (APC) [22]. Recently, tens of thousands of noncoding transcripts have been identified [23]. Many of these non-coding transcripts showed attractive important roles in cancers, which provides new opportunities for the diagnosis and therapy of cancers [24]. A recently identified lncRNA lncRNA-HEIH localizes at chromosome 5q35.3, is polyadenylated, and has 1,681 nucleotides in length [13]. In this study, we further explored the expression, clinical significances, and roles of lncRNA-HEIH in CRC. We found that lncRNA-HEIH is significantly up-regulated in CRC tissues and cell lines, positively associated with tumor size and invasion depth, and indicts poor prognosis of CRC patients. In vitro and in vivo functional experiments demonstrated that enhanced expression of lncRNA-HEIH promotes CRC cell proliferation, decreases CRC cell apoptosis, and promotes CRC tumor growth in vivo. Whereas knockdown of lncRNA$\mathrm{HEIH}$ inhibits CRC cell proliferation, induces CRC cell apoptosis, and suppresses CRC tumor growth in vivo. These data suggest that up-regulated lncRNA-HEIH functions as an oncogene in CRC, and imply that lncRNA-HEIH may be a potential prognostic biomarker and therapeutic target for CRC.

The action mechanisms of lncRNAs are complex. lncRNAs could physically bind to DNA, proteins, mRNAs, or miR-
NAs, and change the expression, localization, and function of the binding partners [11]. Among the diverse action mechanisms, the competitive bindings to miRNAs of lncRNAs are increasingly reported [8]. Through competitively binding common miRNAs, lncRNAs relieve the degradation and/or translation repression of targeted mRNAs caused by the common miRNAs. However, accumulating evidences reveal that, except the roles of miRNAs in the cytoplasm, miRNAs are also found in the nucleus [25]. These nuclear miRNAs could activate or repress gene transcription via binding to promoters, enhancers or transcription factors. In our previous study, we have revealed that miR-939 physically binds to NF- $\kappa \mathrm{B}$, exerts decoy-like actions to prevent the binding between NF- $\mathrm{B}$ and the promoter of its target gene $\mathrm{Bcl}-\mathrm{xL}$, and further represses Bcl-xL transcription [19]. Interestingly, we identified three miR-939 binding sites on lncRNA-HEIH. RNA pull-down assays confirmed the interaction between lncRNA-HEIH and miR-939. RIP and ChIP assays demonstrated that the interaction between lncRNA-HEIH and miR939 damages the binding between miR-939 and NF- $\kappa B$, counteracts the effects of miR-939 on the binding between NF- $\kappa \mathrm{B}$ and Bcl-xL promoter. Luciferase reporter assays, qRTPCR, and western blot showed that the interaction between IncRNA-HEIH and miR-939 counteracts the effects of miR939 on the transcription and expression of Bcl-xL. Furthermore, the expression of Bcl-xL is positively associated with that of lncRNA-HEIH in CRC tissues. Blocking the interac- 
A

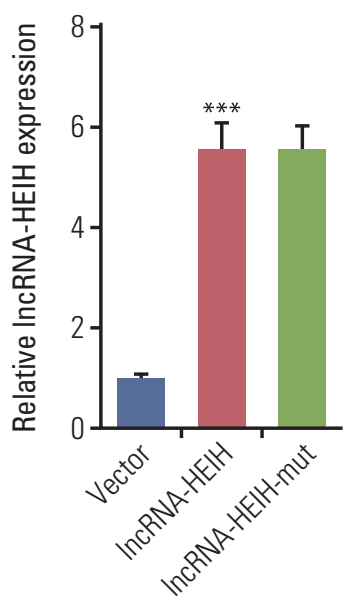

B

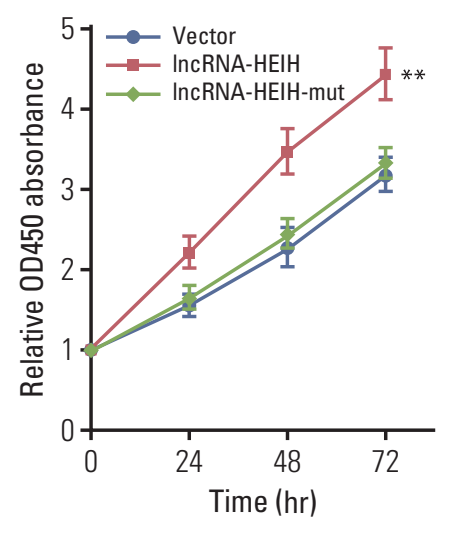

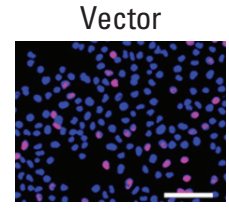

InCRNA-HEIH

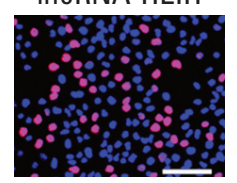

IncRNA-HEIH-mut

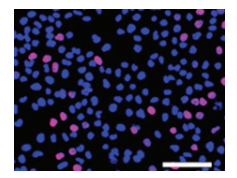

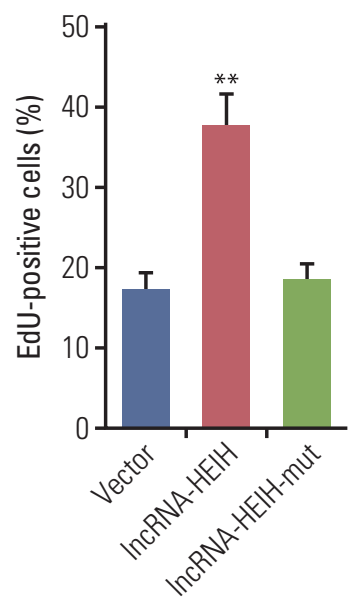

D

$\mathbf{E}$
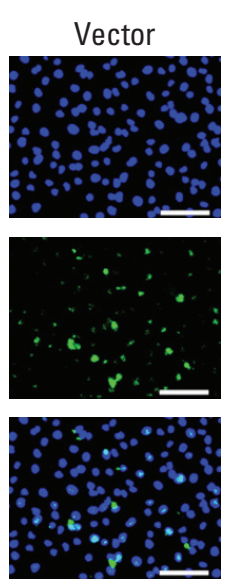
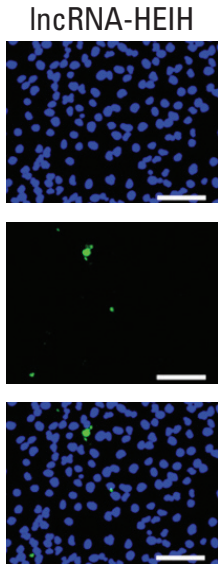
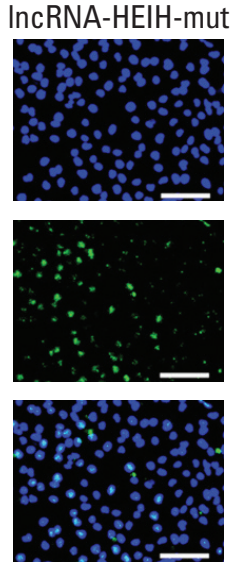
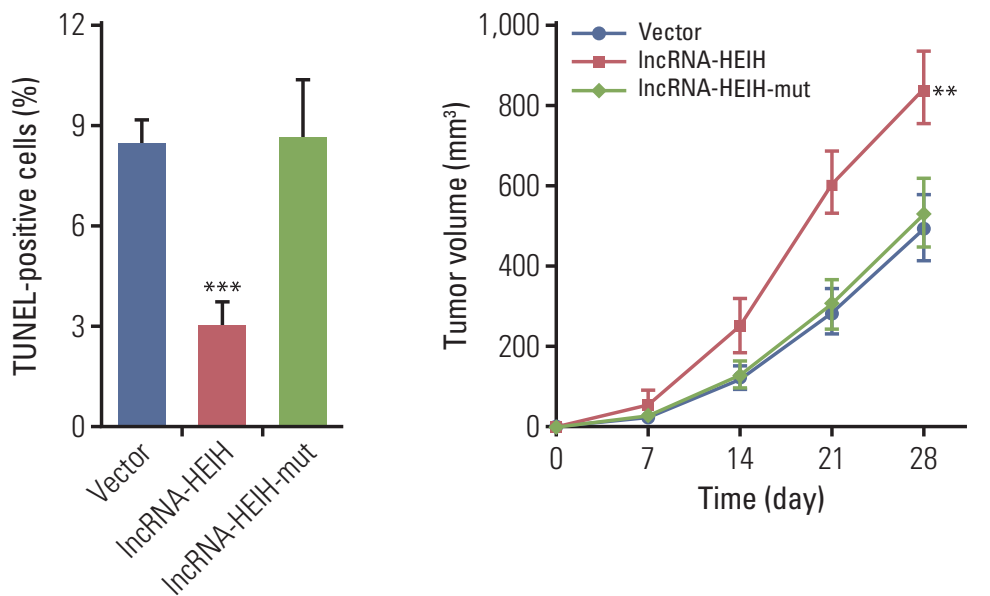

Fig. 7. The mutation of miR-939 binding sites on long noncoding RNA HEIH (lncRNA-HEIH) abolished the effects of IncRNA-HEIH on colorectal cancer tumorigenesis. (A) The expression of lncRNA-HEIH in lncRNA-HEIH or lncRNA-HEIHmut stably overexpressed and control HT-29 cells was detected by quantitative real-time polymerase chain reaction and normalized to glyceraldehyde 3-phosphate dehydrogenase. (B) Cell proliferation rate of lncRNA-HEIH or lncRNA-HEIH-mut stably overexpressed and control HT-29 cells were detected by the Cell Counting Kit-8 assays. (C) Proliferative cells of lncRNA-HEIH or lncRNA-HEIH-mut stably overexpressed and control HT-29 were labeled with ethynyl deoxyuridine (EdU). Red color indicts EdU-positive cells. Scale bars $=100 \mu \mathrm{m}$. (D) The level of apoptosis in IncRNA-HEIH or IncRNAHEIH-mut stably overexpressed and control HT-29 cells was detected by TdT-mediated dUTP nick end labeling (TUNEL) staining. Blue color indicts TUNEL-positive cells. Scale bars $=100 \mu \mathrm{m}$. For A-D, results are shown as mean \pm standard deviation (SD) from three independent experiments. ${ }^{* *} \mathrm{p}<0.01,{ }^{* * *} \mathrm{p}<0.001$ by Student's $\mathrm{t}$ test. (E, F) IncRNA-HEIH or IncRNA-HEIHmut stably overexpressed and control HT-29 cells were subcutaneously injected into nude mice. Tumor volumes were detected every 7 days (E). (Continued to the next page) 
$\mathbf{F}$

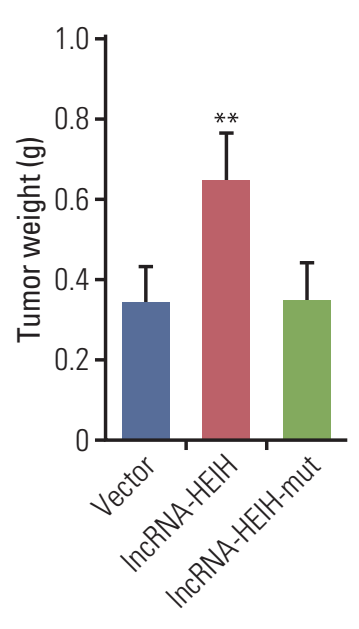

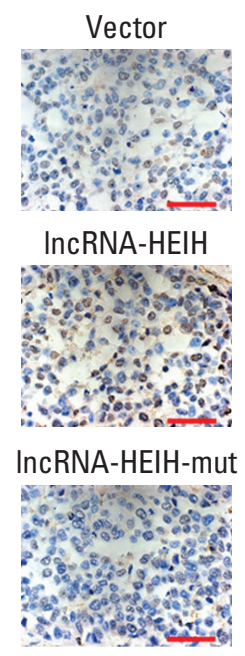

G

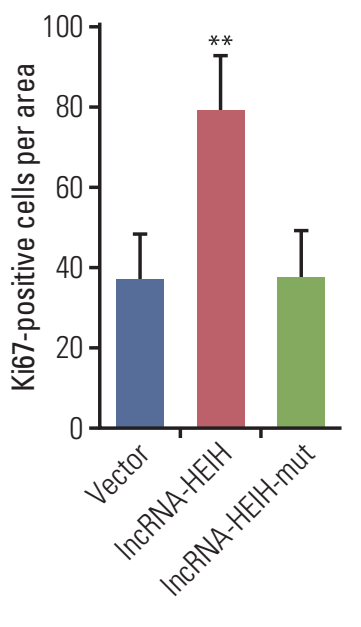

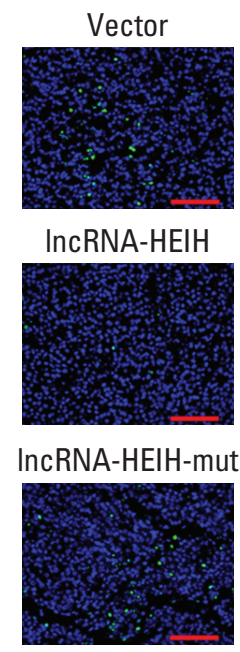

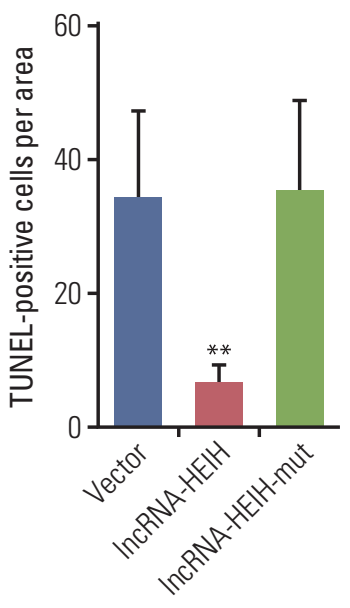

Fig. 7. (Continued from the previous page) Tumor weights were detected at the 28th day after injection (F). (G) Tumors generated from subcutaneous injection with lncRNA-HEIH or lncRNA-HEIH-mut stably overexpressed and control HT-29 cells were immunohistochemistry stained for Ki-67. Scale bars $=50 \mu \mathrm{m}$. (H) The same tumors as in panel G were stained for TUNEL. Scale bars $=100 \mu \mathrm{m}$. For E-H, results are shown as mean \pm standard deviation from six mice. ${ }^{* *} \mathrm{p}<0.01$ by Mann-Whitney $\mathrm{U}$ test.

tion between lncRNA-HEIH and miR-939 by the mutation of miR-939 binding sites on IncRNA-HEIH abolishes the biological effects of IncRNA-HEIH on CRC tumorigenesis. These data suggest that IncRNA-HEIH promotes CRC tumorigenesis through counteracting the roles of miR-939. Except for Bcl-xL, miR-939 has been reported to regulate the expression of SLC34A2, APC2, and VE-cadherin in other cancers [26]. Through binding to miR-939, whether IncRNAHEIH also regulates the expression of these miR-939 targets in CRC needs further investigation. Metastasis is the common reason of CRC patients' death. Whether lncRNA-HEIH regulates the proliferation and/or apoptosis of metastatic CRC cells in a similar manner also needs further investigation. Furthermore, whether there is correlation between IncRNA-HEIH and well-known genes of colon cancer carcinogenesis such as KRAS, p53, and APC is not clear and also needs further investigation.

In conclusion, our study showed that lncRNA-HEIH is up-regulated in CRC tissues and cell lines, associated with tumor size, invasion depth, and poor outcome of CRC patients, promotes CRC cell proliferation and inhibits CRC cell apoptosis in vitro, and promotes CRC tumor growth in vivo via counteracting miR-939-mediated transcriptional repression of $B c l-x L$. Our results suggested that lncRNAHEIH may be a novel diagnostic biomarker and therapeutic target for CRC.

\section{Conflicts of Interest}

Conflict of interest relevant to this article was not reported.

\section{Acknowledgments}

This study was supported by the Guangdong Province Natural Science Foundation (2014A030313295).

\section{References}

1. Torre LA, Bray F, Siegel RL, Ferlay J, Lortet-Tieulent J, Jemal A. Global cancer statistics, 2012. CA Cancer J Clin. 2015;65:87108.
2. He D, Ma L, Feng R, Zhang L, Jiang Y, Zhang Y, et al. Analyzing large-scale samples highlights significant association between rs10411210 polymorphism and colorectal cancer. Bio- 
med Pharmacother. 2015;74:164-8.

3. Levy J, Cacheux W, Bara MA, L'Hermitte A, Lepage P, Fraudeau $\mathrm{M}$, et al. Intestinal inhibition of Atg7 prevents tumour initiation through a microbiome-influenced immune response and suppresses tumour growth. Nat Cell Biol. 2015;17:1062-73

4. Li Y, Liang L, Dai W, Cai G, Xu Y, Li X, et al. Prognostic impact of programed cell death-1 (PD-1) and PD-ligand 1 (PD-L1) expression in cancer cells and tumor infiltrating lymphocytes in colorectal cancer. Mol Cancer. 2016;15:55.

5. Depeille P, Henricks LM, van de Ven RA, Lemmens E, Wang CY, Matli M, et al. RasGRP1 opposes proliferative EGFRSOS1-Ras signals and restricts intestinal epithelial cell growth. Nat Cell Biol. 2015;17:804-15.

6. Markowitz SD, Bertagnolli MM. Molecular origins of cancer: Molecular basis of colorectal cancer. N Engl J Med. 2009;361: 2449-60.

7. Djebali S, Davis CA, Merkel A, Dobin A, Lassmann T, Mortazavi A, et al. Landscape of transcription in human cells. Nature. 2012;489:101-8.

8. Yuan JH, Yang F, Wang F, Ma JZ, Guo YJ, Tao QF, et al. A long noncoding RNA activated by TGF-beta promotes the invasionmetastasis cascade in hepatocellular carcinoma. Cancer Cell. 2014;25:666-81.

9. Mercer TR, Dinger ME, Mattick JS. Long non-coding RNAs: insights into functions. Nat Rev Genet. 2009;10:155-9.

10. Liu X, Xiao ZD, Han L, Zhang J, Lee SW, Wang W, et al. LncRNA NBR2 engages a metabolic checkpoint by regulating AMPK under energy stress. Nat Cell Biol. 2016;18:431-42.

11. Lin A, Li C, Xing Z, Hu Q, Liang K, Han L, et al. The LINK-A lncRNA activates normoxic HIF1alpha signalling in triplenegative breast cancer. Nat Cell Biol. 2016;18:213-24.

12. Rui Q, Xu Z, Yang P, He Z. Long noncoding RNA expression patterns in lymph node metastasis in colorectal cancer by microarray. Biomed Pharmacother. 2015;75:12-8.

13. Yang F, Zhang L, Huo XS, Yuan JH, Xu D, Yuan SX, et al. Long noncoding RNA high expression in hepatocellular carcinoma facilitates tumor growth through enhancer of zeste homolog 2 in humans. Hepatology. 2011;54:1679-89.

14. Bartel DP. MicroRNAs: genomics, biogenesis, mechanism, and function. Cell. 2004;116:281-97.

15. Ge Y, Zhang L, Nikolova M, Reva B, Fuchs E. Strand-specific in vivo screen of cancer-associated miRNAs unveils a role for
miR-21 $\left(^{*}\right)$ in SCC progression. Nat Cell Biol. 2016;18:111-21.

16. Dror S, Sander L, Schwartz H, Sheinboim D, Barzilai A, Dishon $Y$, et al. Melanoma miRNA trafficking controls tumour primary niche formation. Nat Cell Biol. 2016;18:1006-17.

17. Yuan JH, Yang F, Chen BF, Lu Z, Huo XS, Zhou WP, et al. The histone deacetylase 4/SP1 / microrna-200a regulatory network contributes to aberrant histone acetylation in hepatocellular carcinoma. Hepatology. 2011;54:2025-35.

18. Oliverio M, Schmidt E, Mauer J, Baitzel C, Hansmeier N, Khani S, et al. Dicer1-miR-328-Bace1 signalling controls brown adipose tissue differentiation and function. Nat Cell Biol. 2016;18:328-36.

19. Cui C, Yu J, Huang S, Zhu H, Huang Z. Transcriptional regulation of gene expression by microRNAs as endogenous decoys of transcription factors. Cell Physiol Biochem. 2014;33: 1698-714.

20. Chen HC, Kanai M, Inoue-Yamauchi A, Tu HC, Huang Y, Ren $\mathrm{D}$, et al. An interconnected hierarchical model of cell death regulation by the BCL-2 family. Nat Cell Biol. 2015;17:1270-81.

21. Zheng X, Zhang Y, Zhang L, Xu W, Ma W, Sun R, et al. Synergistic inhibition of sunitinib and ethaselen against human colorectal cancer cells proliferation. Biomed Pharmacother. 2016;83:212-20.

22. Feng M, Feng J, Chen W, Wang W, Wu X, Zhang J, et al. Lipocalin2 suppresses metastasis of colorectal cancer by attenuating NF-kappaB-dependent activation of snail and epithelial mesenchymal transition. Mol Cancer. 2016;15:77.

23. Zhang M, Wang W, Li T, Yu X, Zhu Y, Ding F, et al. Long noncoding RNA SNHG1 predicts a poor prognosis and promotes hepatocellular carcinoma tumorigenesis. Biomed Pharmacother. 2016;80:73-9.

24. Zhu XT, Yuan JH, Zhu TT, Li YY, Cheng XY. Long noncoding RNA glypican 3 (GPC3) antisense transcript 1 promotes hepatocellular carcinoma progression via epigenetically activating GPC3. FEBS J. 2016;283:3739-54.

25. Place RF, Li LC, Pookot D, Noonan EJ, Dahiya R. MicroRNA373 induces expression of genes with complementary promoter sequences. Proc Natl Acad Sci U S A. 2008;105:1608-13.

26. Zhang JX, Xu Y, Gao Y, Chen C, Zheng ZS, Yun M, et al. Decreased expression of miR-939 contributes to chemoresistance and metastasis of gastric cancer via dysregulation of SLC34A2 and Raf/MEK/ERK pathway. Mol Cancer. 2017; 16:18. 